\title{
The type and development of Large ratio reducer test bed
}

\author{
Heming Cheng ${ }^{1, a^{*}}$, Tiancun Yang ${ }^{2, b}$, Yanqin Sun ${ }^{3, c}$
}

${ }^{1}$ Mechanical and Electrical Engineering, Huanggang Normal College, Huanggang 438000, China

a184961162@qq.com , b ytcgirl@163.com, c sunyanqin923@163.com

Keywords: Open power flow test bed, Closed power flow test bed, large ratio reducer test bed.

Abstract. In this paper, reducer test bed is structured into two categories from the working principle, open power flow test bed and closed power flow test bed. Analysis of the constituent elements of the gear unit test rig, described the current development situation reducer test bed, summarizes the development direction of the gear unit test bed.

\section{Introduction}

Reducer test bed from the working principle of power flow can be divided into open and closed power flow test bed test bed. Modern electric reducer test bed is moving in the direction of the closed power flow depth development. Closed by electric power flow test bed is easy to control, easy to design and manufacture various components gradually between modular parts, a unit for ease of use and maintenance.

\section{The basic composition of reducer test bed}

The reducer test bed are composed of drive equipment, testing equipment, active reducer, auxiliary reducer, loading equipment (showed in Figure.1). the source power of test bed emitted by a drive device, passing through the detection device, reducer onto the loading device. a motor or an engine is used as a driving device. detecting means for measuring the basic parameters of the process of energy transfer, such as torque, speed, etc. and transfer the parameters to the master device [1]. Generally with torque and speed sensor as a detection device. Generators (AC or DC), hydraulic cylinders,eddy current dynamometer, magnetic brakes,etc. are generally used to load.

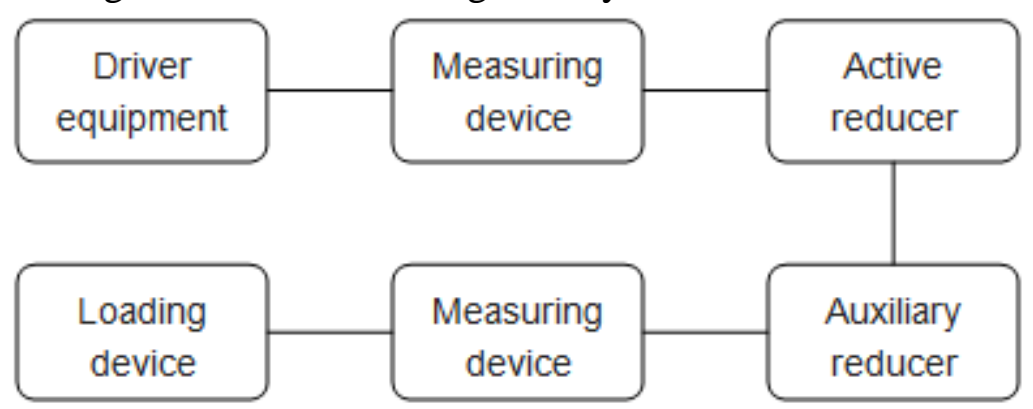

Figure. 1 The basic composition of reducer test bed

\section{The type of reducer test bed}

Generally, reducer test bed is structured into two categories: 1 . open power flow test bed; 2 . closed power flow test bed [2].

\subsection{Open power flow test bed}

Open power flow test bed is a running test bed, which usually compsed of prime mover, subjects reducer and energy load devices. The energy of prime mover is transmitted through the test reducer, final consumption in the load device. Open power flow test bed system diagram shown in Figure.2. 


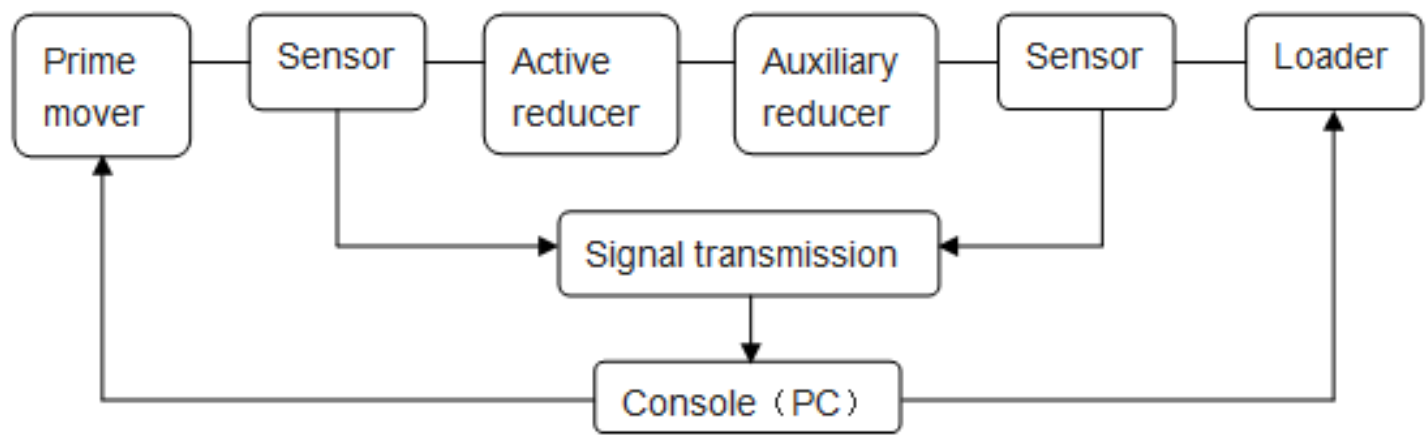

Figure. 2 Open power flow test bed system

The figure shows that the power input test bed system passes through the drive motor, input sensors, active reducer, auxiliary reducer, output sensors. Most of the input power is loss, only part of the energy reaches the loading device and it consumed. The initial investment cost of open power flow test bed is relatively low, the system is easy to maintain, but its drive equipment and loading devices require minimal power is again 1.25 times the rated power of the auxiliary reducer, and the system's input power is completely consumed, and only for a short time operation [3].

\subsection{Closed power flow test bed}

Closed power flow test bed and the open power flow test rig is similar in structure, different places that the closure of power flow test bed loading device having a power recovery function, shown in Figure.3. Different from open power flow test bed is its input power reaches loader is passed energy unit returns to the prime mover, to form a closed power flow. We can see the power of the prime motor output turn full circle back to the original motion partly motor, realized the load, but also saved energy. Lower costs drive device control apparatus closed power flow test bed, the structure is simple, easy to maintain, driving power is only about $30 \%$ of the auxiliary reducer [4]. Closed power flow reducer test bed has a low cost, easy maintenance, energy saving.

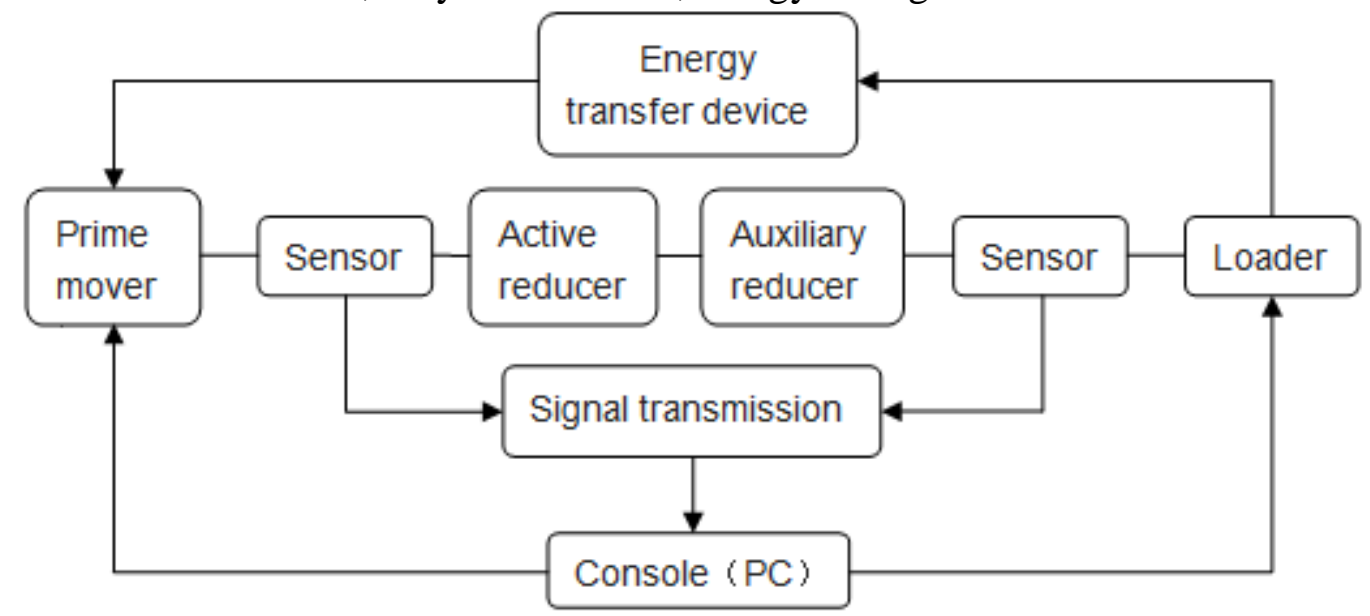

Figure. 3 Closed power flow test bed system diagram

Closed power flow test bed can be divided into mechanical and electrical closing power flow closed power flow two categories. The former is the use for gear drive in energy transfer device, to achieve some mechanical power recycling; the latter is the generator as a loader, powered by magnetic braking torque generated by loading and output of energy feedback, forming a closed system to achieve energy savings. Closed electric power flow test rig according to energy feedback mode can be divided into AC power and DC power back style. AC feedback reducer test bed is driven by $\mathrm{AC}$ motor test, the output torque, speed and other data which from the test bed can be displayed through the computer interface, subjects reducer low speed shaft and accompany the end of the test reducer low speed shaft end by linking shaft connected up, and then accompany the gear unit and driven alternator test run. DC energy feedback reducer test bench is driven by a DC motor reducer test, auxiliary reducer low speed shaft and accompany the end of the low-end of the shaft reducer test 
connected by coupling, reducer and then accompany test drive DC power machine operation. By adjusting the DC excitation current to change the size of the electromagnetic resistance torque, achieving electrical load control [5].

\section{Brief history of large ratio reducer test bed and Research Development}

From the 1960s, there are many scholars have tried to use to build a large ratio planetary gear system test bed, though there was no proven technology to produce relatively complex planetary gear, but such thinking has produced and inspired people. Since the eighties with the great progress of industry, increasing the level of production technology, the application of the planetary gear train more widely, it also will become more in-depth study [6]. Today, high-power test based on planetary gear train is widely used in automobiles, ships, construction machinery, aerospace and military equipment.

We can through a variety of tests and test results to determine the reasonableness of the gear unit design and reliability of its manufacturing, assembly, analyze test results and summarize the reasons that will help assess the mechanical properties of the gear unit, but also for designers provide practical design basis. With the mechanical industry towards high power, high ratio, high efficiency, low energy consumption, low noise and other direction, people put forward higher requirements to reducer. Therefore, further research can effectively detect reducer performance test bed will have a very important significance.

\subsection{Foreign large ratio reduce test bed development history and research status}

Earlier studies conducted abroad on the reducer test bed, such as the United States Gleason company in the 1950s to design a system to make use of wheel load test bed. After some time, Japan's Toyo Seimitsu manufactures a large ship propulsion system with a planetary gear box, which power up to $22,065 \mathrm{KW}$ [7]. Subsequently, many countries put promote research reducer as the main original test bed, relatively well-known USA under the Bureau of Air Research Center Lewis, former Soviet Union machine manufacturing and design institute, the Electrical and Mechanical Engineering, University of Illinois, the United States, Japan's electricity for homes Dynamics, France Skoda company and so the reduction gear test bed has done a lot of research work [8]. Germany was first to open the power flow reducer test bed equipment used in the automotive industry. They designed a lot of gear chamber, after many years of technological development, we realized from the initial test bed design to manufacture and test the final sample have formed a systematic design, manufacturing model [9].

\subsection{Domestic large ratio reducer test bed development history and research status}

Domestic research on gear unit test bed started later than abroad, began in the early 1980's, Licheng Yi compiled the "mechanical drive enclosed test bed" in 1983, which first proposed the concept of a closed power flow. Domestic earlier in this area of research units mainly Zhengzhou Machinery Research Institute, Xi'an Jiaotong University, Sichuan Institute, Chongqing University, Zhangjiakou Coal Mining Machinery factory. They built a decelerator test bed includes various forms of mechanical loading test bed, electrical load test bed in the whole build process has accumulated a wealth of practical experience, has made considerable progress. guixian $\mathrm{Li}$, who designed a mechanical blocking power flow reducer test bench in 2004 [10], and using the test bed to do torsional vibration analysis.Henan University of Science and Technology. Kai xu established by ANSYS finite element analysis software in 2008, the basic unit of body information meshing and simulation analysis, and finally get transmission error results for NEW planetary gear train. Li bao by $2 \mathrm{~K}-\mathrm{H}$ planetary gear train passing efficiency at different power curve analysis in 2009 , gives some good recommendations in the design of the planetary gear. In the same year Taiyuan Coal Research Institute hongjun guo proposed two types of reducer test bed system according to the structure of the classification, he put the open power flow test rig into a closed three kinds of energy feedback type, 
energy type and DC within type [11], the closed power flow test rig divided into mechanical and electrical closed closed two advantages and disadvantages of each type of test bed are analyzed. Currently various reducer test bed for domestic use is widespread dismantling more trouble, the energy consumption of large, variable load loading cumbersome software functions imperfect.

\section{Conclusion}

In test bed type, due to the closed power flow test bed with a simple maintenance, energy and many other advantages, and can be of various grades of gear unit performance testing. Now become mainstream reducer test bed.

In terms of the gear unit test bed loading device, since the DC motor is easy to control, smooth running, power it can be sent back directly to the electric motor, while the use of DC motors simplify the composition of the test bed, reducing the complexity of the system. Therefore, the power flow in a closed trial Taichung widely, play a leading role.

In the reducer test bed control system, in recent years, as electronic technology and modern control theory is widely used in the gear unit test station, we can basically achieve automatic control, such as the use of PC host computer via serial communication indirectly controlled motor start and stop, torque and speed adjustment, brake and other loading equipment, automatic generation and output data reporting features that make traditional reducer test bench function can be improved and perfected [12].

\section{References}

[1] Ishibashi, Akira1, Sonoda, Keiji1, Isami, Shoichi1. Theoretical efficie ncies of planetary.

[2] Gear drives for speed increase and reduction with experimental verification. J. American Society of Mechanical Engineers, 1996,88:397-404.

[3] Hongjun Guo categories reducer test bed performance comparison [J] Mechanical Engineer, 2009, (7): 156-157.

[4] Yibin He, Dingfang Chen, Juan Zhang,Ganwei Cai. The Virtual Design and Assembly of A Multilevel Planetary Reducer. J. The third International Conference 2008, (2):802-806.

[5] Qi Xueting Novel semi-enclosed power flow gear test rig [J] Shandong Institute of Building Materials News, 1996,10 (2): 34-39.

[6] Light rain Huangfu. Reducer Research and Control System test bed. Taiyuan University of Technology master's thesis.

[7] Lai TaShi.Sesign and machining of the epicycloid planet gear of cycloid drives. J. International journal of Adbanced Manufacturing Technology,2006,28(7) :665-670.

[8] Xiao Li, Gui Yinli, Yun Wei. Intersecting axis gear test bed thickening Torsional vibration analysis [J]. Journal of Harbin Institute of Technology, 2004,36 (10), 1405-1406.

[9] Li Xinhua, Gao Chong gold, leather and so on. Gear reducer enclosed test bed loading system [J]. Crane transport machinery, 2009 (5), 48-50.

[10] The mechanical properties of the former pottery Motor Test System [J] Zhejiang: Zhejiang University, 2005, (10): 37-38.

[11] Xiao Liu reducer load test analysis [J] research and analysis, 2008, (10): 39-43.

[12] Hua Wei reducer loading unit test [J] Jianghan Petroleum Technology, 2009,19 (2): 46-48. 